\title{
Dirac Redux - Dirac's Equation in Physical Spacetime without Internal Degrees of Freedom
}

Abstract. The Dirac equation (DE) is one of the cornerstones of quantum physics. We prove in the present contribution that the notion of internal degrees of freedom of the electron represented by Dirac's matrices ${ }^{1,2}$ is superfluous. One can write down a coordinate-free manifestly covariant equation by direct quantization of the energy-momentum 4-vector with modulus $m: \mathrm{P} \psi=m \psi$ (no slash!), the spinor $\psi$ taking care of the different vector grades at the two sides of the equation. Electron spin and all the standard DE properties emerge from this equation. In coordinate representation, the four orthonormal time-space frame vectors $\mathrm{x}_{\mu}$ formally substitute Dirac's $\gamma_{\mu}$-matrices, the two sets obeying to the same Clifford algebra. The present formalism expands Hestenes' spacetime algebra (STA) by adding a reflector vector $\mathrm{x}_{5}$, which in 3D transforms a parity-odd vector $\mathbf{x}$ into a parity-even vector $\boldsymbol{\sigma}=\mathrm{x}_{5} \mathbf{x}$ and vice versa. STA augmented by the reflector will be referred to as STAR, which operates on a real vector space of same dimension as the equivalent real dimension of Dirac's complex $4 \times 4$ matrices. There are no matrices in STAR and the complex character springs from the signature and dimension of spacetime-reflection. This appears most clearly by first showing that STAR comprises two isomorphic subspaces, one for the generators of polar vectors and boosts and the other for the generators of axial vectors and rotors, comprising Pauli spin vectors. These then help to discuss the symmetries, probability current, transformation properties and nonrelativistic approximation of STAR DE. By proving that Dirac's matrices are redundant, because all the information from them is contained in spacetime-reflection, it becomes relevant to reexamine those areas of modern physics that take Dirac matrices and their generalizations as fundamental.

The 92 years old Dirac's equation $(\mathrm{DE})^{1,2}$ is one of the most far-reaching equations in quantum physics: it is compatible with special relativity, the spin of the electron springs from the equation and it predicted the first instance of antiparticle, the positron, discovered four years later by Anderson. ${ }^{3}$ In modern notation DE for a free electron is $(c=1)^{4,5}$

$\left(\gamma^{\mu} p_{\mu}-m\right) \psi=0 \quad$ where: $p_{\mu}=i \hbar \partial_{\mu}=i \hbar \partial / \partial x^{\mu} ; \gamma^{\mu} \cdot \gamma^{\nu}=\eta^{\mu \nu} ; \mu=0,1,2,3$

In (1) $m$ is electron's mass and $\eta^{\mu \nu}=\delta^{\mu v}(1,-1,-1,-1)$ is the time-space signature. Dirac argued that $\gamma^{\mu}$ have to be $4 \times 4$ complex matrices "...describing some new degrees of freedom belonging to some internal 
motion in the electron". ${ }^{1,2}$ This remains the standard position today. ${ }^{4,5}$ Dirac matrices are considered fundamental to DE, truly to any domain of theoretical physics where spin is relevant. ${ }^{4}$

Based on the observation that the Clifford algebra of Dirac matrices is isomorphic to the algebra of spacetime vectors, Hestenes ${ }^{6}$ proposed a new version of DE in the formalism of his space-time algebra STA. ${ }^{7}$ There is no matrix and no imaginary number in STA DE. However, in Hestenes' approach it comes with a price; the spin has to be put 'by hand' in the equation with the spin vector defining a preferred direction, apparently breaking isotropy. The argument against this criticism ${ }^{7}$ is that the spin vector can take any direction and that by convention one chooses a reference direction, much in the same way as one chooses the matrix $\gamma_{0}$ (resp. $\left.\sigma_{3}\right)$ to be diagonal in the Dirac (Pauli) basis. ${ }^{4,5}$ Despite the proven equivalence to the standard DE, apparently, by assuming spin the predictive power of STA DE diminishes compared to standard DE. The real vector space of STA has 16 basis elements, which is half of the equivalent real dimensions for Dirac's $4 \times 4$ complex matrices.

In the present contribution, we demonstrate that it is possible and rewarding to get the best of the standard and STA approaches to DE by taking the most direct path, i.e. by quantizing the relativistic momentum 4vector of modulus $m c^{2}$ (rest energy of the particle). If one could find a suitable algebraic expression, this would make the natural "square root" for the classical scalar invariant $\mathrm{p}^{2} c^{2}=E^{2}-\mathbf{p}^{2} c^{2}=m^{2} c^{4}$. One cannot just equalize a 4-vector $\mathrm{p} c$ to a scalar $m c^{2}$, but if a (wave)function $\psi$ appears to the right of both terms, as it does in quantum mechanics, then it becomes possible, $\psi$ undertaking the duty of equalizer between the vector and scalar degrees. In this sense, the present approach needs the basic form of quantum equations. We will show this in the following after a quick introduction of a Clifford algebra over a real vector space. Then we will present the STAR DE and show a first example of how the algebra plays together with the conjugate pair of position and momentum operators. A systematic investigation of STAR DE will follow by first presenting the formal structure of STAR, then showing how spin springs from momentum, derive the probability current, symmetries and nonrelativistic approximation of DE yielding the Pauli equation. Finally, after conclusions, some important relations and derivations of results in the STAR formalism appear in the Appendix. 
The Clifford or geometric product ${ }^{6,7}$ of two vectors combines Hamilton's symmetric scalar product with Grassmann's antisymmetric wedge product ${ }^{8,9}$; in coordinate-free form it looks like:

$\mathrm{uv}=\mathrm{u} \cdot \mathrm{v}+\mathrm{u} \wedge \mathrm{v} ; 2 \mathrm{u} \cdot \mathrm{v}=(\mathrm{uv}+\mathrm{vu})=\{\mathrm{u}, \mathrm{v}\} ; 2 \mathrm{u} \wedge \mathrm{v}=(\mathrm{uv}-\mathrm{vu})=[\mathrm{u}, \mathrm{v}]$

The two parts are naturally related to the anticommutator and commutator, as shown. The bivector $\mathrm{u} \wedge \mathrm{v}=$ $-v \wedge u$ represents the oriented surface encompassed by the two vectors. The geometric product (2) is linear and invertible, if not zero. It combines a scalar and a bivector, which if normalized make up rotors or boosts, the generators of Lorentz transformations. Examples of both will appear in the following. With the timespace signature $\eta_{\mu \nu}=\delta_{\mu \nu}(1,-1,-1,-1)$ the geometric product of spacetime orthonormal frame vectors is: $\mathrm{x}_{\mu} \mathrm{x}_{\nu} \equiv \mathrm{x}_{\mu \nu}=\mathrm{x}_{\mu} \cdot \mathrm{x}_{v}+\mathrm{x}_{\mu} \wedge \mathrm{x}_{v}=\eta_{\mu \nu}+\mathrm{x}_{\mu} \wedge \mathrm{x}_{v} ; \mathrm{u}=u_{\mu} \mathrm{x}_{\mu} ; \quad \mu, v=0,1,2,3$

Upright letters depict vectors; italics depict scalars. Indices of frame vectors and of scalar components can be lowered or lifted with the help of the signature $\eta_{\mu \nu}=\eta_{\mu}^{\nu}=\eta^{\mu \nu}$; however this feature will be used sparingly where it really becomes useful (see relations $(7,10,11)$ ). The four spacetime frame vectors $\mathrm{x}_{\mu}$ obey the same Clifford algebra as the four Dirac matrices $\gamma_{\mu}$, which is the key observation leading to spacetime algebra, STA. ${ }^{6,7}$ The relativistic 4-momentum vector $\mathrm{p}$ of modulus $m$ and different forms of its square, coordinatefree and coordinate-bound, are given below $(c=1)$ (sum over repeated indices applies by default):

$\mathrm{p}=\mathrm{x}_{\mu} p_{\mu} ; \mathrm{pp}=\mathrm{p} \cdot \mathrm{p}+\mathrm{p} \wedge \mathrm{p}=\mathrm{x}_{\mu \nu} p_{\mu} p_{v}=\eta_{\mu \nu} p_{\mu} p_{v}=\mathrm{p} \cdot \mathrm{p}=m^{2}$

Using same notation for corresponding quantum operators, (4) leads directly to a relativistic quantum vector equation of first order in spacetime derivatives for a particle of mass $m$ ( $I$ is the pseudoscalar, which commutes with all elements of the STAR vector space as shown in the following, see definition (8)):

$\mathrm{p} \psi=m \psi$ with $\mathrm{p}=\hbar I \nabla=\hbar I \mathrm{x}_{\mu} \partial_{\mu} ; \partial_{\mu} \equiv \partial / \partial x^{\mu}=\eta_{\mu}^{\nu} \partial / \partial x_{v} ;$ sum over repeated indices

This is the Dirac equation for a free particle in STAR. STAR is the vector space STA augmented by one independent vector $\mathrm{x}_{5}$, as explained below. The choice of $I$ substituting the algebraic imaginary unit $i \equiv$ $\sqrt{-1}$ is justified in the discussion following equation (11). As already mentioned, the wave function $\psi$ will take care of grade differences between the vectorial quantity $\mathrm{p}$ and the scalar $m . \nabla$ is both coordinate-free and shorthand for $\mathrm{x}_{\mu} \partial_{\mu}$, so there is no need for the Feynman slash notation! $\psi$ must have four components, i.e. it 
is a 4-spinor because of the four spacetime dimensions, as will become clear in the following. If the particle has charge $e$, in the presence of electromagnetic 4-potential A, (5) generalizes to:

$\mathrm{P} \psi=m \psi \quad$ where $\mathrm{P} \equiv \mathrm{p}+e \mathrm{~A}=\hbar I \nabla+e \mathrm{~A}=\mathrm{x}_{\mu}\left(\hbar I \partial_{\mu}+e A_{\mu}\right)$

This is the STAR DE minimally coupled to electromagnetic (EM) field. The two first equations in (5) and (6) are frame-free, which underlines the manifest relativistic covariance of STAR DE, as explained further down by equation (23). The vector-operator $\nabla$ follows the chain rule: $\nabla \mathrm{A}=\mathrm{x}_{\mu \nu} \partial_{\mu} A_{\nu}=\mathrm{x}_{\mu \nu}\left(\partial_{\mu}\left(A_{v}\right)+A_{\nu} \partial_{\mu}\right)$. Therefore, we need to generalize the commutator in (2) as illustrated below for the pair of observables momentum-position (the connection of terms in bold to frame vectors $x_{\mu}$ is evident within the chain of equations below; they depict vectors in 3D as will become clear in (8), (9), e.g. $\left.\boldsymbol{\nabla}=\mathrm{x}_{j 0} \partial_{x_{j}}=\mathbf{x}_{j} \partial / \partial x_{j}\right)$ :

$$
\begin{aligned}
& {[\mathrm{x}, \mathrm{p}]=I \hbar(\mathrm{x} \nabla-\nabla \mathrm{x})=I \hbar \mathrm{x}_{\mu \nu}\left(x_{\mu} \partial_{v}-\partial_{\mu} x_{v}\right)=I \hbar\left[\eta_{\mu \nu} \partial_{\mu}\left(x_{v}\right)+\mathrm{x}_{\mu \nu}\left(x_{\mu} \partial_{v}-x_{v} \partial_{\mu}\right)\right]=I \hbar[4+} \\
& \left.2 \mathrm{x}_{j 0}\left(x_{j} \partial_{0}-x_{0} \partial_{j}\right)+2 \mathrm{x}_{j k}\left(x_{j} \partial_{k}-x_{k} \partial_{j}\right)\right]=I \hbar\left[4+2 \mathbf{x}_{j}\left(x_{0} \partial_{x_{j}}+x_{j} \partial_{0}\right)-2 \epsilon_{j k l} I \boldsymbol{\sigma}_{l}\left(x_{j} \partial_{x_{k}}-x_{k} \partial_{x_{j}}\right)\right]= \\
& I \hbar\left[4+2\left(x_{0} \boldsymbol{\nabla}+\mathbf{x} \partial_{0}\right)-2 I(\boldsymbol{\sigma},(\mathbf{x} \times \nabla))\right]=4 I \hbar\left[1+\mathrm{K}_{j}\left(x_{j} \partial_{t}+t \partial_{x_{j}}\right)+\epsilon_{j k l} J_{l}\left(x_{j} \partial_{x_{k}}-x_{k} \partial_{x_{j}}\right)\right]= \\
& 4 I \hbar[1+\mathbf{K}+\mathbf{J}]
\end{aligned}
$$

Notice index-lifting in action, e.g. $\eta_{\mu \nu} \partial_{\mu}\left(x_{v}\right)=\partial_{\mu}\left(x^{\mu}\right)=4$. Commutator (7) unites dimensionality (cumulative uncertainty relation), boost $\mathbf{K}$ and rotation $\mathbf{J}$ generators with $\mathrm{K}_{j} \equiv \mathrm{x}_{j 0} / 2 \equiv \mathbf{x}_{j} / 2, \mathrm{~J}_{l} \equiv$ $\epsilon_{j k l} \mathrm{x}_{j k} / 2=-\epsilon_{j k l} \mathbf{x}_{j k} / 2=-\epsilon_{j k l} \boldsymbol{\sigma}_{j k} / 2=-I \boldsymbol{\sigma}_{l} / 2$, which together generate proper Lorentz transformations. See (23) and Appendix A) for some basic relations involving these generators. In order to further clarify the different terms in (7) and to prepare for the analysis of DE it is now appropriate to introduce a basis for the real vector space $\mathrm{X}$ and the associated Clifford algebra, together constituting STAR:

$\mathrm{X}:\left\{1(1), \mathrm{x}_{\mu}(4), \mathrm{x}_{\lambda} \mathrm{x}_{\mu} \equiv \mathrm{x}_{\lambda \mu}(6), \mathrm{x}_{\lambda \mu \nu}(4), \mathrm{x}_{0123}(1), \mathrm{x}_{5}(1), \mathrm{x}_{\mu 5}(4), \mathrm{x}_{\lambda \mu 5}(6), \mathrm{x}_{\lambda \mu \nu 5}(4), \mathrm{x}_{01235}=\right.$ $I(1) ; \lambda, \mu, v=0,1,2,3\} ; v \neq \lambda \neq \mu \neq v$. In all 32 real basis elements: $\operatorname{dim} 32$.

From (3) any permutation of given vectors can at most change the sign, which does not add new basis elements. The vector space $\mathrm{X}$ has same real dimension as the equivalent number of real dimensions for Dirac's $4 \times 4$ complex matrices. The first 16 elements in (8) are the same as in STA. ${ }^{7}$ The other 16 elements are obtained by multiplying the STA elements with the 'reflector' $\mathrm{x}_{5}$, which anticommutes with all $\mathrm{x}_{\mu}$. The 
name springs from $x_{5}$ distinguishing parity-odd from parity-even vectors in 3D (see (9)), from its central role in $\mathcal{C}$ - Charge conjugation (particle-antiparticle) and in $\mathcal{C P \mathcal { T }}$ symmetries of DE, (see (20-21)), as well as in defining left and right handed rotors and spinors (see Appendix B). There are five vectors $\left\{\mathrm{x}_{\mu}, \mathrm{x}_{5}\right\}$ generating the basis $(8)$ of the real vector space $\mathrm{X}$, the fifth 'dimension' $\mathrm{x}_{5}$ standing for reflection/handiness. The pentavector (pseudo-scalar) $I$ commutes with all elements in X. Two 3D subspaces are of special interest:

$\mathbf{X}:\left\{1(1), \mathbf{x}_{j} \equiv \mathrm{x}_{j} \mathrm{x}_{0} \equiv \mathrm{x}_{j 0}(3), \mathbf{x}_{j k}(3), \mathbf{x}_{123}(1)\right\} ; \operatorname{dim} 8$

$\boldsymbol{\Sigma}:\left\{1(1), \boldsymbol{\sigma}_{j} \equiv \mathrm{x}_{j 05}=\mathbf{x}_{j} \mathrm{x}_{5}(3), \boldsymbol{\sigma}_{j k}=\mathbf{x}_{j k}=\epsilon_{j k l} I \boldsymbol{\sigma}_{l}(3), \boldsymbol{\sigma}_{123}=\mathbf{x}_{123} \mathrm{x}_{5}=I(1)\right\} ; \operatorname{dim} 8$

${ }^{\natural}$ Similarly to the standard case, ${ }^{4}$ a vector space isomorphic to $(8)$ occurs from the direct product $\mathrm{X}=\boldsymbol{\Sigma} \otimes \boldsymbol{\Sigma}$ with the pseudo-scalar $I=$ $\boldsymbol{\sigma}_{123}$ freely passing through $\otimes$. In addition to providing an alternative route for deriving all the results presented here, this representation marks a further argument in favor of expanding STA to STAR (see also the last equation (35)).

The subspaces $\mathbf{X}, \boldsymbol{\Sigma}$ share their even grade members, i.e. the scalar 1 (grade 0 ) and the three bivectors $\mathbf{x}_{j k}=$ $\boldsymbol{\sigma}_{j k}=\mathrm{x}_{k j}($ grade 2). We will present the parity transformation further down (eq. (16-17)). Here it is sufficient to remember that under such a transformation 3D polar vectors reverse sign and are parity-odd; while axial vectors do not change and are parity-even. The vectors $\mathbf{x}_{j}$ behave as polar vectors (parity-odd), while $\boldsymbol{\sigma}_{j}$ behave as axial vectors (parity-even). The subspace of 'axial' vectors and rotor generators $\boldsymbol{\Sigma}$ is isomorphic to the space of Pauli matrices and their Clifford algebra, ${ }^{7}$ therefore the notation, which in this case follows the tradition in the STA literature. ${ }^{6,710}$ However, in STA the spin vector is 'polar', not 'axial' as here. From (9) it is clear that each vector in one subspace multiplied by $\mathrm{x}_{5}$ produces a corresponding vector in the other subspace. The subspace of 'polar' vectors and boost generators $\mathbf{X}$ is the same as the even subspace of Hestenes' STA, in our notation having $\mathbf{x}_{123}=I \mathrm{x}_{5}$ as 'local' pseudoscalar. ${ }^{6,7,10}$ Unlike STA, the 'global' pseudoscalar I in STAR commutes with all the elements of the vector space X.

The vector $\mathrm{x}_{0}$ is Hermitian, while $\mathrm{x}_{j}$ are anti-Hermitian, as dictated by the signature, therefore $\sigma_{j}$ can be Hermitian if and only if $x_{5}$ is Hermitian:

$\left\{\mathrm{x}_{\mu}^{\dagger}=\eta_{\mu}^{v} \mathrm{x}_{v}=\mathrm{x}^{\mu} ; \mathrm{x}_{\lambda \mu \nu}^{\dagger}=\mathrm{x}_{v}^{\dagger} \mathrm{x}_{\mu}^{\dagger} \mathrm{x}_{\lambda}^{\dagger}=\mathrm{x}^{v \mu \lambda} ; \mathrm{x}_{5}^{\dagger}=\mathrm{x}_{5}\right\} \Rightarrow \mathrm{x}_{j}^{\dagger}=\mathrm{x}_{j} ; \boldsymbol{\sigma}_{j}^{\dagger}=\sigma_{j} ; I^{\dagger}=-I$

The operation of reversing a multivector $\widetilde{\mathrm{x}_{\lambda \mu \nu}}=\mathrm{x}_{\nu \mu \lambda}$ corresponds to taking the transpose of a matrix; by the way, $\tilde{I}=I$. The most general rule for obtaining the Hermite conjugate in STAR is to take the reverse and 
swap the position of each index from up (down) to down (up), as illustrated for the trivector in (10). The operations of reversion $(\sim)$ and contravariant - covariant index swapping $(\downarrow \uparrow)$ commute:

$\mathrm{A}^{\dagger}=(\widetilde{\mathrm{A}(\downarrow \uparrow)})=\widetilde{\mathrm{A}}(\downarrow \uparrow)$, e.g. $\mathrm{x}_{\tau}=\zeta_{\tau \omega} \mathrm{x}^{\omega} ;\left(\mathrm{x}^{\tau} \mathrm{x}_{\omega}\right)^{\dagger}=\mathrm{x}^{\omega} \mathrm{x}_{\tau}$ with $\zeta_{\tau \omega} \equiv \delta_{\tau \omega}(+---+)=\zeta^{\tau \omega} ; \tau, \omega=$

$0,1,2,3,5$. The signature $\zeta_{\tau \omega}$ expands timespace signature $\eta_{\mu \nu}$ by the Hermitian reflector.

In the context of STAR the properties (10) are the result of the spacetime-reflection signature $\zeta_{\tau \omega}$ and of the number of total dimensions (five). The same signature and the odd number of space dimensions enforce $I^{2}=$ -1 . There are 16 basis elements in (8) squaring to -1 and that can and do generate a complex structure; of these it is only the oriented pentavolume element $I$ that by containing all the generating vectors of $\mathrm{X}$, is the oriented basis element of maximal isotropy. In addition, it commutes with all elements of X. For these reasons, it makes the obvious choice to replace the algebraic imaginary unit $i$, as already done in (5-7). Other choices, let say a bivector $\sigma_{j k}=\mathrm{x}_{k j}=\epsilon_{j k l} I \boldsymbol{\sigma}_{l}$ does indeed square to -1 , but is clearly anisotropic.

Alike STA, STAR promotes a geometric view of physics, where vectors and their combinations, not the scalar components set the complex structure. With the above preliminaries in place, it is instructive to calculate at first the square of the STAR DE (6):

$\mathrm{P} \psi=m \psi \Rightarrow \mathrm{PP} \psi=m^{2} \psi \Rightarrow\left(\eta_{\mu \nu}\left(\hbar I \partial_{\mu}+e A_{\mu}\right)\left(\hbar I \partial_{v}+e A_{v}\right)-m^{2}+e \hbar I(\nabla \wedge \mathrm{A}+\mathrm{A} \wedge \nabla)\right) \psi=$ $\left(\mathrm{KG}+e \hbar I\left(-\boldsymbol{\nabla}\left(A_{0}\right)-\partial_{0}(\mathbf{A})+I \mathrm{x}_{5}(\boldsymbol{\nabla} \times \mathbf{A})\right)\right) \psi=\left(\mathrm{KG}+e \hbar I\left(\mathbf{E}+I \mathrm{x}_{5} \mathbf{B}\right)\right) \psi \equiv(\mathrm{KG}+e \hbar I(\mathbf{E}+$

$I(\boldsymbol{\sigma}, \mathbf{B}))) \psi \equiv(\mathrm{KG}+e \hbar I \mathrm{~F}) \psi=0$

$\mathbf{A}=A_{j} \mathbf{x}_{j}$ (vector potential); $\mathbf{E}=E_{j} \mathbf{x}_{j}$ (electric field, polar $3 \mathrm{D}$ vector); $I(\boldsymbol{\sigma}, \mathbf{B}) \equiv I \boldsymbol{\sigma}_{j} B_{j}=$ $I \mathrm{x}_{5} \mathbf{B}$ (magnetic field, axial 3D (bi)vector); $\mathrm{F} \equiv \mathbf{E}+I(\boldsymbol{\sigma}, \mathbf{B})$ (Faraday)

KG stands for Klein-Gordon term; it comprises only scalar and pseudoscalar components, including operators. The term $\mathbf{E}+I \mathrm{x}_{5} \mathbf{B}=\mathbf{E}+I(\boldsymbol{\sigma}, \mathbf{B})$ is independent of $\mathrm{x}_{5}$ and is also known as the Faraday $\mathrm{F}$. It depicts the relativistic invariant EM field strength experienced by the electron, as marked by the prefactor $e \hbar I . \mathrm{F}$ is a tensor in the standard formalism; ${ }^{4,10}$ as in the case of STA, in STAR it is a 4D bivector. ${ }^{7,10}$ The term $e \hbar I \mathrm{~F}$ distinguishes the squared $\mathrm{DE}$ from the $\mathrm{KG}$ equation. It represents internal degrees of freedom of the electron - the spin, interacting with the EM field. Indeed, in the nonrelativistic regime equation (12) (or 
the Pauli equation (31)) yields equation (13) below, as shown in Appendix C (orbital and spin angular momentum vectors: $\mathbf{L} \equiv \mathbf{r} \times \mathbf{p} ; \mathbf{S}=\hbar \boldsymbol{\sigma} / 2 ; \varphi$ is a Pauli spinor approximating Dirac's spinor $\psi$ in (12)):

$$
\left(\mathrm{P} \cdot \mathrm{P}-m^{2}+e \hbar I \mathrm{~F}\right) \psi=0 \stackrel{\text { nonrelativistic approx. }}{=}\left[I \hbar \partial_{t}-\frac{\mathbf{p}^{2}}{2 m}+e A_{0}-\frac{e}{2 m} \mathbf{B} \cdot(\mathbf{L}+2 \mathbf{S})\right] \varphi=0
$$

This is the famous prediction from DE that the unit of spin angular momentum interacts twice as strongly with the magnetic field as the unit of orbital angular momentum. The derivation of (12-13) proves that spin springs from 4-momentum in spacetime-reflection, without any preconceived internal degrees of freedom. The spin magnetic moment of the electron from (13) is $-\hbar e \sigma / 2 m c$ that in absolute value is a factor of $\sim 1.00118$ smaller than the experimental figure, the gap arising from QED multiparticle effects beyond $\mathrm{DE} .{ }^{4,5}$ As a second illustration, let us find the conserved current ${ }^{2,4}$ of DE in STAR. From (10) $\mathbf{x}_{j}^{\dagger}=\mathbf{x}_{j}$, which simplifies the derivation of the Hermite conjugate for DE and of the Dirac spinor conjugate $\bar{\psi}$ :

$$
\begin{aligned}
& (\mathrm{P}-m) \psi=0 \Rightarrow\left(\mathrm{x}_{\mu}\left(\hbar I \partial_{\mu}+e A_{\mu}\right)-m\right) \psi=\left(\left(\hbar I \partial_{0}+e A_{0}\right)+\mathrm{x}_{j}\left(\hbar I \partial_{j}+e A_{j}\right)-m \mathrm{x}_{0}\right) \mathrm{x}_{0} \psi \\
& \stackrel{\dagger}{\Rightarrow} \psi^{\dagger} \mathrm{x}_{0}\left(\left(-\hbar I \partial_{0}+e A_{0}\right)+\mathrm{x}_{j}\left(-\hbar I \partial_{j}+e A_{j}\right)-m \mathrm{x}_{0}\right) \equiv \bar{\psi}\left(\mathrm{x}_{\mu}\left(-\hbar I \partial_{\mu}+e A_{\mu}\right)-m\right) \mathrm{x}_{0}=0
\end{aligned}
$$

After Hermite conjugation $\dagger, \partial_{\mu}$ act to the left. Left-multiplying the DE by $\bar{\psi}$ and right-multiplying the last equation in (14) by $\bar{\psi}^{\dagger}=\mathrm{x}_{0} \psi$ then subtracting it from the first, one obtains the conservation of probability current (the angled brackets below take the scalar part of the product inside):

$$
\left(\partial_{\mu} \bar{\psi}\right) \mathrm{x}_{\mu} \psi+\bar{\psi} \mathrm{x}_{\mu}\left(\partial_{\mu} \psi\right)=\partial_{\mu}\left(\bar{\psi} \mathrm{x}_{\mu} \psi\right)=0 \text { with probability density } \rho=\left\langle\bar{\psi} \mathrm{x}_{0} \psi\right\rangle=\left\langle\psi^{\dagger} \psi\right\rangle \geq 0
$$

We will look more closely to the use of angled brackets ${ }^{6,7}$, the values of the Lorentz scalar $\langle\bar{\psi} \psi\rangle$ and the probability density $\rho$ after having specified a form for the spinor $\psi$ in STAR, see (29), (33).

Third, let us look at the symmetries parity, time reversal and charge conjugation for the STAR DE and for the spinor $\psi$. Transformations of vectors appear as two-sided operations, while for spinors as one-sided.

Parity $\mathcal{P}: \mathrm{x}_{\mu} \rightarrow \eta_{\mu}^{v} \mathrm{x}_{\nu}=\mathrm{x}_{0} \mathrm{x}_{\nu} \mathrm{x}_{0}$. As in the standard treatment left-multiplying DE by $\mathrm{x}_{0}$ we obtain:

$$
\left\{\mathrm{x}_{0}\left(\mathrm{x}_{\mu} P_{\mu}-m\right) \psi=\left(\eta_{\mu}^{v} \mathrm{x}_{v} P_{\mu}-m\right) \mathrm{x}_{0} \psi \equiv\left(\eta_{\mu}^{v} \mathrm{x}_{v} P_{\mu}-m\right) \psi_{\mathcal{P}}\right\} \Rightarrow \psi_{\mathcal{P}}=\mathrm{x}_{0} \psi
$$

Applying the parity transformation (16) to the 3D vectors from (9) we clearly see that: 
$\mathbf{x}_{j} \stackrel{\mathcal{P}}{\rightarrow} \mathrm{x}_{0} \mathbf{x}_{j} \mathrm{x}_{0}=-\mathbf{x}_{j}$ is polar, while $\boldsymbol{\sigma}_{j} \stackrel{\mathcal{P}}{\rightarrow} \mathrm{x}_{0} \boldsymbol{\sigma}_{j} \mathrm{x}_{0}=\boldsymbol{\sigma}_{j}$ is axial

Time reversal $\mathcal{T}: \mathrm{x}_{\mu} \rightarrow-\eta{ }_{\mu}^{v} \mathrm{x}_{v}=\left(I \mathrm{x}_{0}\right) \mathrm{x}_{v}\left(I \mathrm{x}_{0}\right)=\mathrm{x}_{1235} \mathrm{x}_{v} \mathrm{x}_{1235}$, which is an antiunitary transformation that can be also seen as a tetravector or tetravolume reflection. Hermite conjugating DE (see (14)) then reversing we obtain (below $\hbar=1$ ):

$\left[\bar{\psi}\left(\mathrm{x}_{\mu}\left(-I \overleftarrow{\partial}_{\mu}+e A_{\mu}\right)-m\right) \mathrm{x}_{0}=0\right]^{\sim}=\mathrm{x}_{0}\left(\mathrm{x}_{\mu}\left(-I \partial_{\mu}+e A_{\mu}\right)-m\right) \widetilde{\bar{\psi}}=\left(\mathrm{x}_{0}\left(I \partial_{0}^{\prime}+e A_{0}\right)+\mathrm{x}_{j}\left(I \partial_{j}-e A_{j}\right)-\right.$

$m) \mathrm{x}_{0} \widetilde{\bar{\psi}}=0 \Rightarrow \psi_{\mathcal{T}}=\mathrm{x}_{0} \widetilde{\bar{\psi}}=\widetilde{\psi^{\dagger}}$

We can also look at the effect of $\mathcal{T}$ on the Dirac Hamiltonian:

$H=\hbar I \partial_{t}=-e A_{0}-\hbar I \boldsymbol{\nabla}+e \mathbf{A}+m \mathrm{x}_{0} \stackrel{\mathcal{J}}{\rightarrow} H_{T}=\hbar I \partial_{0}^{\prime}=\hbar I \partial_{-t}=-e A_{0}+\mathrm{x}_{j 0}\left(\hbar I \partial_{j}-e A_{j}\right)+m \mathrm{x}_{0}=$

$-e A_{0}-\hbar I \boldsymbol{\nabla}-e \mathbf{A}+m \mathrm{x}_{0}$. Notice that $\stackrel{\mathcal{A}}{\rightarrow}-\mathbf{A}$ as it should.

Charge conjugation $\mathcal{C}: e \rightarrow-e$. As above Hermite conjugate and reverse; then left-multiply by $\mathrm{x}_{213}$ :

$\left[\bar{\psi}\left(\mathrm{x}_{\mu}\left(-I \overleftarrow{\partial}_{\mu}+e A_{\mu}\right)-m\right) \mathrm{x}_{0}=0\right]^{\sim}=\mathrm{x}_{0}\left(-\mathrm{x}_{\mu}\left(I \partial_{\mu}-e A_{\mu}\right)-m\right) \widetilde{\bar{\psi}} \stackrel{\mathrm{x}_{213}(}{\longrightarrow}\left(\mathrm{x}_{\mu}\left(I \partial_{\mu}-e A_{\mu}\right)-m\right) \mathrm{x}_{0123} \widetilde{\bar{\psi}} \Rightarrow$

$\psi_{\mathcal{C}}=\mathrm{x}_{0123} \widetilde{\bar{\psi}}=I \mathrm{x}_{5} \widetilde{\bar{\psi}}$

I in the last term adds an overall phase and can be dropped. If DE (6) describes the electron, then (20)

describes its antiparticle, the positron. The result of the three symmetries in STAR applied onto DE is:

$\mathcal{C P} \mathcal{T}: \mathrm{x}_{\mu} \rightarrow \mathrm{x}_{5} \mathrm{x}_{\mu} \mathrm{x}_{5}=-\mathrm{x}_{\mu}$. Applying the three operations in series yields $(\mathcal{C P} \mathcal{P}) \mathrm{DE}:\left(\mathrm{x}_{\mu}\left(I \partial_{\mu}+e A_{\mu}\right)-\right.$ $m) \underset{\mathcal{T}}{\rightarrow}\left(\mathrm{x}_{0}\left(I \partial_{0}^{\prime}+e A_{0}\right)+\mathrm{x}_{j}\left(I \partial_{j}-e A_{j}\right)-m\right) \mathrm{x}_{0} \underset{\widetilde{\psi}}{\stackrel{\mathcal{P}}{\rightarrow}}\left(\mathrm{x}_{0}\left(I \partial_{0}^{\prime}+e A_{0}\right)-\mathrm{x}_{j}\left(I \partial_{j}-e A_{j}\right)-\right.$

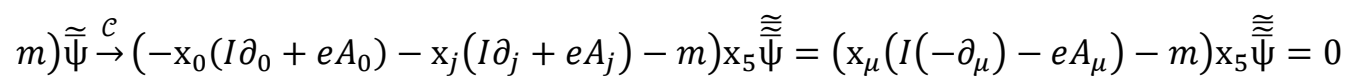

Compared to DE both charge and 4-momentum have reversed in (21). Left-multiplication of DE by $\mathrm{x}_{5}$ yields the same transformation of the Dirac operator as in (21), therefore within an overall phase:

$\psi_{\mathcal{C P \mathcal { T }}}=\mathrm{x}_{5} \approx \overline{\widetilde{\psi}}_{\mathrm{\psi}}=\mathrm{x}_{5} \psi$

One can also show independently that $\cong \widetilde{\widetilde{\psi}}=\widetilde{\widetilde{\psi}}=\psi$ by remembering that $\bar{\psi}=\psi^{\dagger} x_{0}$ and by the definition (11) of Hermite conjugate in STAR. 
Notice that the derivation of symmetries above treats the spacetime frame vectors on equal footing, signature taken into account, which is compatible with the relativity principle; it does not rely on any particular representation that happens to comprise complex and real components as is the case in the standard approach. $^{4}$

Now we will touch shortly the relativistic covariance of the STAR DE (6). The operator $(\mathrm{P}-m)$ is coordinate-free and relativistic-invariant, i.e. it remains unchanged as a whole under a Lorentz transformation $\mathcal{L}$. On the other end, the spinor $\psi$ transformation has to be a one-sided generalized rotor S, i.e. a rotor and/or a boost comprising the exponentiated generators $\mathrm{J}_{j}, \mathrm{~K}_{j}$ we met in (7) with the respective rotation angle and/or rapidity from the transformation $\mathcal{L}$ at hand. Therefore, we can write:

$\underset{\mathcal{L}}{\stackrel{\mathcal{L}}{\rightarrow}} \mathrm{S} \psi \Rightarrow(\mathrm{P}-m) \psi=0 \stackrel{\mathcal{L}}{\rightarrow}(\mathrm{P}-m) \mathrm{S} \psi=0 \Leftrightarrow \mathrm{S}(\mathrm{P}-m) \mathrm{S}^{-1} \mathrm{~S} \psi=\mathrm{S}[(\mathrm{P}-m) \psi]=0$, where $\mathrm{S}=e^{\mathrm{S}_{j} \omega_{j}}$ with $\left\{\begin{array}{c}\mathrm{S}_{j}=\mathrm{K}_{j}=\mathbf{x}_{j} / 2 ; \omega_{j}=\alpha_{j} \text { (rapidity, hyperbolic angle) for boosts } \\ \mathrm{S}_{j}=\mathrm{J}_{j}=-I \boldsymbol{\sigma}_{j} / 2 ; \omega_{j}=\vartheta_{j} \text { (Euclidian angle) for rotors }\end{array}\right.$

The whole STAR DE transforms as a 4-spinor, which it better does as due to spacetime dimensions (four) it is equivalent to four coupled equations that form..., yes, a spinor (see discussion following (33')). The twosided rotor around the operator in $\mathrm{S}(\mathrm{P}-m) \mathrm{S}^{-1} \mathrm{~S} \psi=0$ is useful for calculating the transformed frame vectors and components of 4-momentum and 4-potential, if needed as discussed in the next paragraph. The simplicity of (23) (compare with the linearized infinitesimal Lorentz transformations in the standard approach! $)^{2,4,5}$ makes it meaningful to state that the relativistic covariance of the STAR DE is manifest. Compared to standard DE, STAR DE stands at an advantage point when infinitesimal Lorentz transformations become essential, like in curved spacetime. ${ }^{4}$

How do we change basis in STAR? (23) contains the answer: by two sided general rotor transformations, i.e. $\mathrm{x}_{\mu} \rightarrow \mathrm{x}_{\mu}^{\prime}=\mathrm{Sx}_{\mu} \mathrm{S}^{-1}$. One can generalize the rotor to also comprise $\mathrm{x}_{5}$. For example, in the deep relativistic regime $(|\mathrm{p}| \gg m)$ one can neglect the mass term in DE and adapt a more convenient basis and corresponding spinor than the STAR Dirac basis adapted here, known as the Weyl basis and Weyl spinors. This is done by applying the generalized rotor $R_{\omega}=e^{\mathrm{x}_{50} \omega / 2}$ one-sided to spinors and two-sided to vectors, which can swap $\mathrm{x}_{0}$ with $\mathrm{x}_{5}$ leaving $\mathrm{x}_{j}$ unchanged: $R_{\omega} \mathrm{x}_{j} R_{\omega}^{-1}=\mathrm{x}_{j}$, as recounted in Appendix $\mathrm{B}$. The symmetries above help us specify a form for the spinor $\psi$ in STAR expressed as a couple of Pauli spinors as now shown. 
The strategy consists in finding a form of DE that is entirely expressed in the basis of subspace $\boldsymbol{\Sigma}$ in (9), i.e. make disappear any explicit dependence from $\mathrm{x}_{0}, \mathrm{x}_{5}$ by, as in the standard procedure, splitting the Dirac spinor $\psi$ into two Pauli spinors $\varphi, \chi \cdot{ }^{2,4-6}$ By inspection of DE as written below at the form $(\boldsymbol{\Sigma})$, it becomes clear that in addition to $\varphi, \chi$ the form of $\psi$ we are looking for has to depend explicitly only on $\mathrm{x}_{0}$ and $\mathrm{x}_{5}$ (below $\left.P_{j} \mathrm{x}_{j}=P_{j} \mathbf{x}_{j} \mathrm{x}_{0}=\mathbf{P x}_{0}=\mathbf{P x}_{5} \mathrm{x}_{5} \mathrm{x}_{0}=P_{j} \boldsymbol{\sigma}_{j} \mathrm{x}_{50} \equiv(\boldsymbol{\sigma}, \mathbf{P}) \mathrm{x}_{50}\right)$ :

$\mathrm{DE}:\left(\mathrm{x}_{\mu} P_{\mu}-m\right) \psi \stackrel{(\mathbf{X})}{=}\left(P_{0} \mathrm{x}_{0}+\mathbf{P x}_{0}-m\right) \psi \stackrel{(\boldsymbol{\Sigma})}{=}\left(P_{0} \mathrm{x}_{0}+\mathrm{x}_{50}(\boldsymbol{\sigma}, \mathbf{P})-m\right) \psi=0 ; \psi=\psi\left(\mathrm{x}_{0}, \mathrm{x}_{5}, \varphi, \chi\right)$

An alternative form of $\mathrm{DE}$ in (24) is to write it together with the parity transformed $\mathcal{P}(\mathrm{DE})$ as follows:

$\mathrm{DE}=\frac{1}{2}[\mathrm{DE}+\mathcal{P}(\mathrm{DE})+\mathrm{DE}-\mathcal{P}(\mathrm{DE})]=\frac{1}{2}\left[\left(1+\mathrm{x}_{0}\right) \mathrm{DE}+\left(1-\mathrm{x}_{0}\right) \mathrm{DE}\right] \stackrel{(\mathbf{X})}{\Rightarrow} \frac{1}{2}\left[\left(P_{0}-m+\mathbf{P}\right)\left(1+\mathrm{x}_{0}\right)-\right.$

$\left.\left(P_{0}+m+\mathbf{P}\right)\left(1-\mathbf{x}_{0}\right)\right] \psi \stackrel{(\boldsymbol{\Sigma})}{=} \frac{1}{2}\left[\left(P_{0}-m+\mathbf{x}_{5}(\boldsymbol{\sigma}, \mathbf{P})\right)\left(1+\mathrm{x}_{0}\right)-\left(P_{0}+m+\mathbf{x}_{5}(\boldsymbol{\sigma}, \mathbf{P})\right)\left(1-\mathbf{x}_{0}\right)\right] \psi=0(25)$

The two projectors $\left(1 \pm \mathrm{x}_{0}\right)$ are orthogonal to each other. From the second last term we choose provisionally $\left(1+\mathrm{x}_{0}\right) \psi \sim \varphi ;\left(1-\mathrm{x}_{0}\right) \psi \sim \chi$. From the last form in (25), in order to be able to remove the $\mathrm{x}_{5}$ factor from the $\mathrm{x}_{5}(\boldsymbol{\sigma}, \mathbf{P})$ term we need to specify the provisional form to, e.g. $\left(1+\mathrm{x}_{0}\right) \psi \sim \varphi ;\left(1-\mathrm{x}_{0}\right) \psi \sim \mathrm{x}_{5} \chi$.

Projectors being idempotent within constant factors (therefore $\sim$ is used instead of $=$ ), they can spread from one side onto both sides of an equation, e.g. $\left(1+\mathrm{x}_{0}\right) \psi \sim \varphi \Rightarrow\left(1+\mathrm{x}_{0}\right) \psi \sim\left(1+\mathrm{x}_{0}\right) \varphi$, so that we can write:

$\psi \sim\left(1+\mathrm{x}_{0}\right) \psi+\left(1-\mathrm{x}_{0}\right) \psi \sim \rho\left(\left(1+\mathrm{x}_{0}\right) \varphi+\left(1-\mathrm{x}_{0}\right) \mathrm{x}_{5} \chi\right)=\rho\left(\varphi+\mathrm{x}_{5} \chi\right)\left(1+\mathrm{x}_{0}\right)$

In (26) $\rho$ is a (pseudo)scalar and the more compact form at the right follows because $\mathrm{x}_{0}$ commutes with the Pauli spinors and anti-commutes with $\mathrm{x}_{5}$. Applying one of the last two forms in (26) to the last equation in (25), or directly to the DE in (24):

$\mathrm{DE}:\left(P_{0} \mathrm{x}_{0}+\mathrm{x}_{50}(\boldsymbol{\sigma}, \mathbf{P})-m\right) \psi=0 \stackrel{(26)}{\Longrightarrow}\left\{\left[\left(P_{0}-m\right) \varphi-(\boldsymbol{\sigma}, \mathbf{P}) \chi\right]+\mathrm{x}_{5}\left[\left(-P_{0}-m\right) \chi+(\boldsymbol{\sigma}, \mathbf{P}) \varphi\right]\right\} \rho\left(1+\mathrm{x}_{0}\right)=$

The quantity inside curly brackets cannot be proportional to $\left(1-\mathrm{x}_{0}\right)$, because apart from $\mathrm{x}_{5}$ all terms in it belong to the subspace $\boldsymbol{\Sigma}$. Therefore, it has to be zero. Due to independence of the two terms ( 1 and $\mathrm{x}_{5}$ are orthonormal basis elements in (8)), (27) is then equivalent to the standard-looking pair of coupled equations below, entirely written in the subspace $\boldsymbol{\Sigma}$ : 
$\left\{\begin{array}{l}\left(P_{0}-m\right) \rho \varphi-(\boldsymbol{\sigma}, \mathbf{P}) \rho \chi=0 \\ \left(P_{0}+m\right) \rho \chi-(\boldsymbol{\sigma}, \mathbf{P}) \rho \varphi=0\end{array}\right.$

A nice feature of projectors in (26) is that you don't need to warry about their Lorentz transformations, because the transformation applies to the whole DE (operator and spinor), and when engaged to the DE the projectors in (26) either annihilate or are absorbed into projectors from the operator part. This property is most clearly seen when applying (26) to the form (25) of DE.

Had we chosen the projector $\left(1-x_{0}\right)$ in the final form of $\psi$ in (26), it would have corresponded to the exchange $\varphi \rightleftarrows \chi$ in (28), which also occurs by applying the $\mathcal{C P \mathcal { T }}$ symmetry (21) $\Psi_{\mathcal{C P \mathcal { T }}}=\mathrm{x}_{5} \psi$ to the 4-spinor $\psi: \rho\left(\varphi+\mathrm{x}_{5} \chi\right)=\psi \rightleftarrows \mathrm{x}_{5} \psi=\rho\left(\chi+\mathrm{x}_{5} \varphi\right)$. Same changes arising from projectors containing $\mathrm{x}_{0}$ and from left multiplication by $\mathrm{x}_{5}$ relates again to symmetries of DE and will be discussed elsewhere. Now, from (27) we calculate the Lorentz scalar $\langle\bar{\psi} \psi\rangle$ and the probability density $\left\langle\psi^{\dagger} \psi\right\rangle$ from (15), showing how to extract the scalar part by use of the angled brackets, and at the same time casting new light onto the role of projectors in STAR spinors.

$\left\{\bar{\psi}=\psi^{\dagger} \mathrm{x}_{0}=\rho^{\dagger}\left(1+\mathrm{x}_{0}\right)\left(\varphi^{\dagger}+\mathrm{x}_{5} \chi^{\dagger}\right) \mathrm{x}_{0}=\rho^{\dagger}\left(1+\mathrm{x}_{0}\right)\left(\varphi^{\dagger}-\mathrm{x}_{5} \chi^{\dagger}\right)\right\} \Rightarrow\left\{\begin{array}{c}\left\langle\psi^{\dagger} \psi\right\rangle \\ \langle\bar{\psi} \psi\rangle\end{array}=\rho^{\dagger} \rho\left\langle\left(1+\mathrm{x}_{0}\right)\left(\varphi^{\dagger} \pm\right.\right.\right.$

$\left.\left.\mathrm{x}_{5} \chi^{\dagger}\right)\left(\varphi+\mathrm{x}_{5} \chi\right)\left(1+\mathrm{x}_{0}\right)\right\rangle=2 \rho^{\dagger} \rho\left\langle\varphi^{\dagger} \varphi \pm \chi^{\dagger} \chi\right\rangle \sim \rho^{2}\left\langle\varphi^{\dagger} \varphi \pm \chi^{\dagger} \chi\right\rangle$

Apart from a normalization factor, the last expression in (29) correctly yields real numbers, non-negative in the case of probability density (+ sign). The angled brackets in (29) will disappear in (33). The projectors in (29) are essential to projecting out the cross-product terms comprising $x_{5}$. However, when transformations of the whole DE are at stake, like Lorentz transformations (23), we already showed that the projectors of the spinor (26) either are absorbed or annihilated into the operator in (25). In these cases the spinor behaves as if it did not comprise projectors. This flexibility of projectors in STAR spinors is powerful and fascinating.

As last illustration of the workings of STAR we derive the Pauli equation ${ }^{11}$ as non-relativistic approximation to STAR DE in (28) (see also Appendix C). We adopt a procedure from Feynman consisting in first isolating the fast oscillating part of $\psi$ in the factor $\rho=\rho(t)$, then cancelling it after the action of $p_{0}=I \hbar \partial_{t}$ :

$\left\{\begin{array}{l}\left(P_{0}-m\right) \rho \varphi-(\boldsymbol{\sigma}, \mathbf{P}) \rho \chi=0 \rho=e^{-I m t / \hbar} \\ \left(P_{0}+m\right) \rho \chi-(\boldsymbol{\sigma}, \mathbf{P}) \rho \varphi=0\end{array}\left\{\begin{array}{c}\left(I \hbar \partial_{t}+e A_{0}\right) \varphi-(\boldsymbol{\sigma}, \mathbf{P}) \chi=0 \\ \left(I \hbar \partial_{t}+e A_{0}+2 m\right) \chi-(\boldsymbol{\sigma}, \mathbf{P}) \varphi=0\end{array}\right.\right.$ 
For $\left|\left(I \hbar \partial_{t}+e A_{0}\right) \chi\right| \ll 2 m|\chi|$ (nonrelativistic regime) the lower of the two coupled equations approximates in lowest order to: $\chi \approx(\boldsymbol{\sigma}, \mathbf{P}) \varphi / 2 m$. I.e. for slow electrons $|\chi| \ll|\varphi|$. Substituting into the upper equation one obtains the Pauli Hamiltonian $H_{P}$ (below: $\mathbf{P}^{2} \equiv(\mathbf{p}+e \mathbf{A}) \cdot(\mathbf{p}+e \mathbf{A})=(-\hbar(I) \boldsymbol{\nabla}+e \mathbf{A})$. $(-\hbar(I) \boldsymbol{\nabla}+e \mathbf{A})$ is a (pseudo) scalar operator):

$I \hbar \partial_{t} \varphi=H_{P} \varphi=\left(\frac{\mathbf{P}^{2}}{2 m}-e A_{0}+\frac{\hbar e}{2 m}(\mathbf{\sigma}, \mathbf{B})\right) \varphi$

This is the STAR Pauli equation (PE), which looks formally identical to the standard $\mathrm{PE},{ }^{2}$ but here without any matrices and with a complex structure surging from the real vector space $(9)$ ! The term $\frac{\hbar e}{2 m c}(\boldsymbol{\sigma}, \mathbf{B})-$, which we met earlier in eq. (13) marks the additional potential energy due to the spin magnetic moment of a slow electron. Of course, eq. (13) can also be derived from PE. The Stern-Gerlach term distinguishes PE from the Schrödinger equation ${ }^{12}$, which is obtained from (31) by removing it (no spin) and by freezing the spinor $\varphi$, let say to spin up. In analogy to the case of DE we can now express the Pauli spinor $\varphi$ as function of the two spin wavefunctions $\varphi_{u}, \varphi_{d}$ :

$\varphi=\rho_{\varphi}\left(\varphi_{u}+\sigma_{1} \varphi_{d}\right)\left(1+\sigma_{3}\right)=\rho_{\varphi}\left(\varphi_{u}-I \sigma_{2} \varphi_{d}\right)\left(1+\sigma_{3}\right)=\rho_{\varphi} R_{\varphi}\left(1+\sigma_{3}\right) ; R_{\varphi}=e^{-I \sigma_{2} \vartheta_{\varphi} / 2}$

The form of spinor with a rotor $R_{\varphi}$ comprising the probability amplitudes for spin up and spin down $\cos (\vartheta / 2)=\varphi_{u} ; \sin (\vartheta / 2)=\varphi_{d}$, allows to treat a number of systems more directly ${ }^{7}$ than by first substituting (32) into (31) then solving the resulting pair of coupled equations. At the same time, one keeps a clear physical picture in the process, which relates to the geometric objects of STA and STAR, like the rotor in (32). The other Pauli spinor $\chi$ takes the same form as $\varphi$ in (32):

$\chi=\rho_{\chi}\left(\chi_{u}-I \sigma_{2} \chi_{d}\right)\left(1+\sigma_{3}\right)=\rho_{\chi} R_{\chi}\left(1+\sigma_{3}\right) ; R_{\chi}=e^{-I \sigma_{2} \vartheta_{\chi} / 2}$

The form of the two Pauli spinors in (32-32') is independent of the nonrelativistic approximation adopted to derive the PE (30). By substituting (32-32') into the couple of equations to the left in (30) (i.e. prior to the nonrelativistic approximation) one can write down four coupled first order equations in terms of $\varphi_{u}, \varphi_{d}, \chi_{u}, \chi_{d}$ and with the $\mathrm{x}_{\mu}$ absent; these are equivalent to STAR DE. The rotor form allows also to express very efficiently many quantities related to DE, again by maintaining a clear geometric picture along 
the way. Examples from this report comprise the Dirac probability currents (15) and the symmetry transformed DE in (16-21), or scalar quantities in (29). Let us look at these last:

$\left\langle\varphi^{\dagger} \varphi \pm \chi^{\dagger} \chi\right\rangle=\rho_{\varphi}^{2} \pm \rho_{\chi}^{2}$

The scalar quantities in (33) are the square moduli of the two Pauli spinors. Finally, the similarity between the first equalities in (32-32') and the Dirac spinor (26) is not a coincidence. For the first, one can write (26) as a generalized rotor (of the same type as the one transforming Dirac's to Weyl's basis! See equation (8A) in Appendix B):

$\psi=\rho\left(\varphi+\mathrm{x}_{5} \chi\right)\left(1+\mathrm{x}_{0}\right)=\rho R_{\omega}^{\prime}\left(1+\mathrm{x}_{0}\right) ; R_{\omega}^{\prime}=e^{\mathrm{x}_{50} \omega \prime / 2}=\cos \frac{\omega \prime}{2}+\mathrm{x}_{50} \sin \frac{\omega \prime}{2}=\varphi+\mathrm{x}_{50} \chi$

(As a side spring, in (34) $\omega^{\prime}=0$ corresponds to the particle at rest; only the Pauli spinor $\varphi$ appears in the rest frame, which makes sense as the electron has only two spin degrees of freedom. The case $\omega^{\prime}=\pi / 2$ is discussed in eq. (8A) in Appendix). For the second, there is a symmetry underlying the transition from Dirac's equation (6), written in the full basis $\mathrm{X}$ in (8) to its equivalent form as a pair of coupled equations in (28), written in Pauli's subspace $\boldsymbol{\Sigma}$ from (9). The symmetry becomes apparent by the isomorphism between X and the direct product $\boldsymbol{\Sigma} \otimes \boldsymbol{\Sigma}, \mathrm{X} \leftrightarrow \boldsymbol{\Sigma} \otimes \boldsymbol{\Sigma}$, mentioned in the note of definition (9). From the correspondence:

$\mathrm{x}_{0} \leftrightarrow 1 \otimes \sigma_{3} ; \mathrm{x}_{5} \leftrightarrow 1 \otimes \sigma_{1} ; \mathrm{x}_{50} \leftrightarrow 1 \otimes\left(-I \sigma_{2}\right)$

it becomes clear that what we have achieved by going from (6) to (28) and from (26) to (32-32') is just to reverse the direct products (35)! A kind of reflection, which encapsulates the necessity of $x_{5} \leftrightarrow 1 \otimes \sigma_{1}$.

In conclusion, the definition of STAR DE shows its clear physical and geometrical meaning as the most direct quantization of the classical 4-momentum vector with modulus $m$. Its demonstrated working hints to the expectation that all the formal machinery developed in nine decades to handle DE and its generalizations, adapt easily to the STAR formalism. In many instances, results are achieved more directly than in standard $\mathrm{DE}$, as shown for the Lorentz covariance in (23). The present contribution demonstrates that the information from $\mathrm{DE} \gamma$-matrices is contained in spacetime-reflection; therefore, it becomes relevant to question the role of the same matrices in areas of modern physics considering them as fundamental. The STAR formalism comprises STA, and together with its inborn distinction between 3D polar and axial vectors, it can affect the 
formalism of many areas of physics. In our description of nature, by developing Dirac's ideas we come to recognize handiness/reflection as fundamental, on a par with space and time.

\section{Appendix.}

A) More about rotor and boost generators. The commutators of boost and rotor generators are:

$$
\begin{aligned}
& \left\{\mathrm{J}_{j} \equiv \frac{1}{2} \epsilon_{j}^{k l} \mathrm{x}_{k l}=-\frac{1}{2} I \boldsymbol{\sigma}_{j} \text { (Rotors); } \mathrm{K}_{j} \equiv \frac{1}{2} \mathrm{x}_{j 0}=\frac{1}{2} \mathbf{x}_{j}=\frac{1}{2} \mathrm{x}_{5} \boldsymbol{\sigma}_{j} \text { (Boosts) }\right\} \Rightarrow\left\{\left[\mathrm{J}_{j}, \mathrm{~J}_{k}\right]=-\frac{1}{2} \boldsymbol{\sigma}_{j k}=\right. \\
& \left.-\frac{1}{2} \epsilon_{j k l} I \boldsymbol{\sigma}_{l}=\epsilon_{j k l} \mathrm{~J}_{l} ;\left[\mathrm{K}_{j}, \mathrm{~K}_{k}\right]=\frac{1}{2} \mathbf{x}_{j k}=-\epsilon_{j k l} \mathrm{~J}_{l} ;\left[\mathrm{J}_{j}, \mathrm{~K}_{k}\right]=-\frac{1}{2} I \mathrm{x}_{5} \mathbf{x}_{j k}=\frac{1}{2} \epsilon_{j k l} \mathrm{x}_{5} \mathbf{x}_{l}=\epsilon_{j k l} \mathrm{~K}_{l}\right\}
\end{aligned}
$$

By the definition of $\mathrm{J}_{j}, \mathrm{~K}_{j}$ in (1A) it is clear that they do not comprise $\mathrm{x}_{5}$. The rotor-boost space can split into right and left handed disjoint subspaces, $\mathrm{S}_{+j}$ and $\mathrm{S}_{-j}$, illustrating the double coverage of the groups underlying 3D and 2D spinor spaces $S O(1,3)=S U(2) \otimes S U(2)$ :

$\left\{\mathrm{S}_{ \pm j} \equiv \frac{1}{2}\left(\mathrm{~J}_{j} \pm I \mathrm{~K}_{j}\right)=\frac{1}{2} \mathrm{~J}_{j}\left(1 \mp \mathrm{x}_{5}\right)\right\} \Rightarrow\left\{\left[\mathrm{S}_{ \pm j}, \mathrm{~S}_{ \pm k}\right]=\frac{1}{4} \epsilon_{j k l} \mathrm{~J}_{l}\left(1 \mp \mathrm{x}_{5}\right)^{2}=\frac{1}{2} \epsilon_{j k l} \mathrm{~J}_{l}\left(1 \mp \mathrm{x}_{5}\right)=\right.$

$\left.\epsilon_{j k l} \mathrm{~S}_{ \pm l} ;\left[\mathrm{S}_{+j}, \mathrm{~S}_{-k}\right]=0\right\}$

Notice the entrance with $I$ of $\mathrm{x}_{5}$ into $\mathrm{S}_{ \pm j}$. In STAR rotors and boosts acting one-sided onto a Dirac spinor constitute proper Lorentz transformations for the spinor as shown in eq. (23) in the main text and here:

$\psi \stackrel{\mathcal{L}}{\rightarrow} \mathrm{S} \psi=e^{\mathrm{S}_{j} \omega_{j}} \psi$, with $\left\{\begin{array}{c}\mathrm{S}_{j}=\mathrm{K}_{j} ; \omega_{j}=\alpha_{j} \text { (rapidity) for boosts } \\ \mathrm{S}_{j}=\mathrm{J}_{j} ; \omega_{j}=\vartheta_{j} \text { (angle) for rotors }\end{array}\right.$

B) Weyl spinors. Similarly to (2A), the orthonormal projectors $\left(1 \pm \mathrm{x}_{5}\right) / 2$ separate Dirac spinors into rightand left-handed spinors, known as Weyl spinors:

$\psi=\psi_{L}+\psi_{R}=\frac{1}{2}\left(1-\mathrm{x}_{5}\right) \psi+\frac{1}{2}\left(1+\mathrm{x}_{5}\right) \psi$

Let see where this form might be useful. The Dirac spinor and basis are preferred to describe slow electrons.

For fast electrons, in conditions $\mathrm{p} \gg m$, we can neglect the mass term (of course, we enter here a regime where the validity of DE becomes questionable due to possibility of particle - antiparticle creation and annihilation); DE in momentum representation takes the form: 
$(\mathrm{p}-m) \psi(\mathrm{p})=0 \stackrel{\mathrm{p} \gg m}{\longrightarrow} \mathrm{p} \psi(\mathrm{p}) \approx 0$

If $\psi(p)$ is a solution to the DE at the r.h.s. then $x_{5} \psi(p)$ will also be a solution, $x_{5}$ anticommuting with all $x_{\mu}$. Now, proceeding in the same way as we did to recover the form of the Dirac spinor in (26), we write (5A) as:

$\frac{1}{2}\left[\rho\left(1+\mathrm{x}_{5}\right) \psi(\mathrm{p})+\rho\left(1-\mathrm{x}_{5}\right) \psi(\mathrm{p})\right]$

By precisely the same reasoning as applied to obtain (26), obviously with the exchange $\mathrm{x}_{0} \rightleftarrows \mathrm{x}_{5}$, we can write down the following form, comprising the Weyl spinors in (4A):

$\psi \stackrel{(4 \mathrm{~A})}{=} \psi_{R}+\psi_{L}=\rho\left[\left(1+\mathrm{x}_{5}\right)\left(\varphi+\mathrm{x}_{0} \chi\right)+\left(1-\mathrm{x}_{5}\right)\left(\varphi+\mathrm{x}_{0} \chi\right)\right]=\rho\left(\varphi+\mathrm{x}_{0} \chi\right)\left(1+\mathrm{x}_{5}\right)$

This form of the spinor is convenient to describe fast electrons or massless fermions. The compact form at the right in $(7 \mathrm{~A})$ is possible because $\mathrm{x}_{5}$ commutates with the Pauli spinors and anticommutes with $\mathrm{x}_{0}$. The swap $\mathrm{x}_{0} \rightleftarrows \mathrm{x}_{5}$ constitutes the passage from the Dirac to Weyl basis. As mentioned in the main text in the discussion following eq. (23) the change of basis in STAR is achieved by two-sided generalized rotor transformations. Let illustrate it here in relation to the passage from Dirac to Weyl basis. It takes the form:

$\left\{\mathrm{x}_{\tau}\right\} \rightarrow\left\{\mathrm{x}_{\tau}^{\prime}\right\}: \mathrm{x}_{\tau}^{\prime}=R_{\omega} \mathrm{x}_{\tau} R_{\omega}^{-1}=e^{\mathrm{x}_{50} \omega / 2} \mathrm{x}_{\tau} e^{-\mathrm{x}_{50} \omega / 2} \stackrel{\omega=\pi / 2}{=}\left\{\begin{array}{c}\mathrm{x}_{0}^{\prime}=\mathrm{x}_{5} \\ \mathrm{x}_{j}^{\prime}=\mathrm{x}_{j} ; \tau=0,1,2,3,5 ; j=1,2,3 \\ \mathrm{x}_{5}^{\prime}=-\mathrm{x}_{0}\end{array}\right.$

Within a sign (not relevant for a basis element) we have realized by $(8 \mathrm{~A})$ the mentioned transformation.

Notice that $x_{50}^{2}=-1$, which makes exponentiation in (8A) meaningful.

C) Derivation of Eq. (13). For convenience, let us rewrite the squared DE (12) here:

$(\mathrm{KG}+e \hbar I(\mathbf{E}+I(\boldsymbol{\sigma}, \mathbf{B}))) \psi=0$

If $\psi=\rho\left(\varphi+\mathrm{x}_{5} \chi\right)$ is a solution to DE then it is also a solution to its square, eq. (9A). We have removed the projectors $\left(1 \pm x_{0}\right)$ from the spinor, because there is no free $x_{0}$ in $(9 A)$. Now we follow the same strategy as earlier and try to write (9A) completely in the $\boldsymbol{\Sigma}$ subspace with the help of the two Pauli spinors:

$$
\begin{aligned}
& \psi=\rho\left(\varphi+\mathrm{x}_{5} \chi\right) \stackrel{(9 \mathrm{~A})}{\Longrightarrow}\left(\mathrm{KG}+e \hbar I\left(\mathrm{x}_{5}(\boldsymbol{\sigma}, \mathbf{E})+I(\boldsymbol{\sigma}, \mathbf{B})\right)\right) \rho\left(\varphi+\mathrm{x}_{5} \chi\right)= \\
& \left\{\begin{array}{l}
{[\mathrm{KG}-e \hbar(\boldsymbol{\sigma}, \mathbf{B})] \rho \varphi+e \hbar I(\boldsymbol{\sigma}, \mathbf{E}) \rho \chi=0} \\
{[\mathrm{KG}-e \hbar(\boldsymbol{\sigma}, \mathbf{B})] \rho \chi+e \hbar I(\boldsymbol{\sigma}, \mathbf{E}) \rho \varphi=0}
\end{array}\right.
\end{aligned}
$$


The coupled equations (10A) are a good starting point for investigating the interaction of both $\mathbf{E}$ and $\mathbf{B}$ fields with the electron. As pointed out by Hestenes, Dirac just ignored the $\mathbf{E}$ term in (9A) under the unjustified excuse that it is imaginary. This will be the subject of future work. Here we take the nonrelativistic limit of (10A). Under the derivation of the Pauli equation we saw that in the nonrelativistic approximation $|\chi| \ll|\varphi|$. This is expected, as by writing DE in the rest frame of the electron in the momentum representation one finds $\chi=0$. Therefore, in this regime we can take $\psi \approx \rho \varphi$ and the two equations in (10A) decouple:

$\left\{\begin{array}{c}{[\mathrm{KG}-e \hbar(\boldsymbol{\sigma}, \mathbf{B})] \rho \varphi=0} \\ e \hbar I(\boldsymbol{\sigma}, \mathbf{E}) \rho \varphi=0\end{array}\right.$

In the following we will look only at the top equation. As done earlier we isolate the fast oscillations in $\rho=$ $e^{-I m t / \hbar}$, so that second derivative $\partial_{t}^{2} \varphi$ of $\varphi$ from the KG term can be ignored after differentiating $\rho$ and removing the exponential. We also assume a weak constant EM field and in this way can also ignore the $\mathrm{A}^{2}$ terms. These simplifications will make treatment easier allowing to focus on few essential properties of DE:

$$
\begin{aligned}
& \left(\mathrm{P} \cdot \mathrm{P}-m^{2}-e \hbar(\boldsymbol{\sigma}, \mathbf{B})\right) \rho \varphi=\left(-\hbar^{2} \partial_{t}^{2}-\mathbf{p}^{2}+e^{2}\left(A_{0}^{2}-\mathbf{A}^{2}\right)+e \hbar I\left(2 A_{0} \partial_{0}+\partial_{0}\left(A_{0}\right)-2 A_{j} \partial_{j}-\partial_{j}\left(A_{j}\right)\right)-\right. \\
& \left.m^{2}-e \hbar(\boldsymbol{\sigma}, \mathbf{B})\right) \exp (-I m t / \hbar) \varphi \approx \exp (-I m t / \hbar)\left(-\hbar^{2}\left(-2 \frac{I m}{\hbar} \frac{\partial \varphi}{\partial t}-\frac{m^{2}}{\hbar^{2}}+\frac{\partial^{2} \varphi}{\partial t^{2}}\right)-\mathbf{p}^{2}+\right. \\
& \left.e \hbar I\left(-2 A_{0} \frac{I m}{\hbar}+\partial_{t}\left(A_{0}\right)-2 A_{j} \partial_{j}-\partial_{j}\left(A_{j}\right)\right)-m^{2}-e \hbar(\boldsymbol{\sigma}, \mathbf{B})\right) \varphi \Rightarrow\left\{I \hbar \partial_{t}-\frac{\mathbf{p}^{2}}{2 m}+e A_{0}-\right. \\
& \left.\frac{e \hbar I}{2 m}\left[2 A_{j} \partial_{j}+\partial_{j}\left(A_{j}\right)\right]-\frac{e \hbar}{2 m}(\boldsymbol{\sigma}, \mathbf{B})\right\} \varphi \approx 0
\end{aligned}
$$

The last equation is closely related and can be derived also from the Pauli equation (31) in the main text. Now, we know that the Schrödinger equation can handle magnetic orbital momentum, which must be the term with square brackets in (12A). Let us render it explicit by trying to express $A_{j}$ as a function of the components of the magnetic field $B_{k}$. Start with $\mathbf{B}=\boldsymbol{\nabla} \times \mathbf{A} \Rightarrow B_{j}=\partial_{j+1}\left(A_{j+2}\right)-\partial_{j+2}\left(A_{j+1}\right)$ with indices varying cyclically mod3, e.g. $B_{2}=\partial_{3} A_{1}-\partial_{1} A_{3}$. The brackets in e.g. $\partial_{j+2}\left(A_{j+1}\right)$ mean that the derivative operates only to that term. Taking $A_{j}=\left(B_{j+1} x_{j+2}-B_{j+2} x_{j+1}\right) / 2$, or $2 \mathbf{A}=\mathbf{B} \times \mathbf{x}$ satisfies the equation for $\mathbf{B}$, remembering that the $B_{j}$ are constant. With this choice the term $\partial_{j}\left(A_{j}\right)=0$ in $(12 \mathrm{~A})$ and $2 \hbar I A_{j} \partial_{j}=2 \mathbf{A}$. $\mathbf{p}=\mathbf{B} \times \mathbf{x} \cdot \mathbf{p}=\mathbf{B} \cdot \mathbf{x} \times \mathbf{p} \equiv \mathbf{B} \cdot \mathbf{L}$, where $\mathbf{L}=\mathbf{x} \times \mathbf{p}$ is the orbital angular momentum. The spin vector being $\mathbf{S}=\hbar \boldsymbol{\sigma} / 2$, we put it all together into the last equation in (12A) and obtain eq. (13) from the main text: 
$\left[I \hbar \partial_{t}-\frac{\mathbf{p}^{2}}{2 m}+e A_{0}-\frac{e}{2 m} \mathbf{B} \cdot(\mathbf{L}+2 \mathbf{S})\right] \varphi=0$

\section{References}

${ }^{1}$ Dirac, P. A. M. The quantum theory of the electron. Proc. Roy. Soc. Lon. A, 117, 610 (1928).

${ }^{2}$ Dirac, P. A. M. Principles of Quantum Mechanics: International Series of Monographs on Physics $4^{\text {th }}$ ed., Ch. XI (Oxford University Press, Oxford, 1958).

${ }^{3}$ Anderson, C. D. The Positive Electron. Phys. Rev. 43, 491 (1933).

${ }^{4}$ Zee, A. Quantum Field Theory in a Nutshell, Part II, III, VIII, (Princeton University Press, $2^{\text {nd }}$ ed. 2010).

${ }^{5}$ Schwabl, F. Advanced Quantum Mechanics, Ch. 15 (Springer-Verlag, Berlin, Heidelberg, New York, $2^{\text {nd }}$ ed. 2004).

${ }^{6}$ Hestenes, D. Space-Time Algebra (Gordon and Breach, New York, 1966).

${ }^{7}$ Doran, C. \& Lasenby, A. Geometric Algebra for Physicists, Ch. 8, (Cambridge University Press, Cambridge 2007).

${ }^{8}$ Grassmann, H. Die Ausdehnungslehre. (Enslin, Berlin, 1862).

${ }^{9}$ Clifford, W. K. Applications of Grassmann's extensive algebra. Am. J. Math., 1, 350 (1878).

${ }^{10}$ Dressel, J., Bliokh, K., Nori, F. Spacetime algebra as a powerful tool for electromagnetism, Physics Reports 589, 1-71 (2015).

${ }^{11}$ Pauli, W. Zur Quantenmechanik des magnetischen Elektrons. Zeitschrift für Physik, 43, 601 (1927).

${ }^{12}$ Schrödinger, E. An Undulatory Theory of the Mechanics of Atoms and Molecules. Phys. Rev., 28, 1049-1070 (1926)

Competing interests: The authors declare no competing interests. 